\title{
Evolving Model for the Complex Traffic and Transportation Network Considering Self-Growth Situation
}

\author{
Wei Zhang and Di Xu \\ School of Management, Xiamen University, Xiamen 361005, China \\ Correspondence should be addressed to Di Xu, dxu@xmu.edu.cn
}

Received 4 May 2012; Revised 29 June 2012; Accepted 13 July 2012

Academic Editor: Wuhong Wang

Copyright (C) 2012 W. Zhang and D. Xu. This is an open access article distributed under the Creative Commons Attribution License, which permits unrestricted use, distribution, and reproduction in any medium, provided the original work is properly cited.

\begin{abstract}
It has been approved that the scale-free feature exists in various complex networks, such as the internet, the cell or the biological networks. In order to analyze the influence of the selfgrowth phenomenon during the growth on the structure of traffic and transportation network, we formulated an evolving model. Based on the evolving model, we prove in mathematics that, even that the self-growth situation happened, the traffic and transportation network owns the scale-free feature due to that the node degree follows a power-law distribution. A real traffic and transportation network, China domestic airline network is tested to consolidate our conclusions. We find that the airline network has a node degree distribution equivalent to the power-law of which the estimated scaling parameter is about 3.0. Moreover the standard error of the estimated scaling parameter changes according to the self-growth probability. Our findings could provide useful information for determining the optimal structure or status of the traffic and transportation network.
\end{abstract}

\section{Introduction}

The study of the coevolution of the dynamics and the topology of transportation networks is a promising research topic, especially for the potential implications in infrastructures planning. Triggered by two invaluable papers [1,2], relevant problems of the complex network have attracted a great deal of attention in the latest decade. Commonly cited examples cover various types of networks such as the information network [3], the social network [4], the communication network [5] and the traffic and transportation network [6-8]. Within the framework of the reference of [1], the scale-free network follows the power-law degree distribution. Several researchers discussed the scale-free characteristic of the traffic and transportation network, including the urban street network [3], the logistics network [9], 
the transit network [10], and the airline network [6]. Using the static model, it was reported that the load distribution over traffic and transportation follows a power law [11, 12]. However, the previous works have done lots of assumptions and simplifications on the structure of traffic and transportation networks, such that there should be only one link between two nodes on the network [1,13]. As it is known to all, the situation not only does exist, but also can be seen commonly that two nodes are connected by more than one link on the traffic and transportation network. For the street network of a city, we could easily imagine that there are many links connecting between one pair of nodes. If we use the adjacent matrix [6] to demonstrate the connecting degree between the two cities, the cell number in the matrix would apparently larger than one. However, these situations were not permitted in the previous researches of the complex network theory for the traffic and transportation network $[1,4,13]$. Furthermore, the evolution of the traffic and transportation network always has the "self-growth" characteristic. For example, the growth of the airline network always undergoes two ways. One way is to connect the exiting airport to the new constructed airport (the new added node) with new airlines. The other one is to open new airlines between the existing airports without any new airport constructed. The latter way of the evolution of the air network expresses the "self-growth" feature. Generally, the relevant decision makers of the traffic and the transportation departments frequently face the problems of opening new lines to service the exiting stations (or the airport, depots and, etc.) or constructing a new station or intersection when the network is expanded. The self-growth characteristic of the traffic and transportation network has not been elaborately described or dealt with in the existing literatures about the scale-free network.

It is true that the dynamics in the network adapts to the topology, but, on the other side, topology of the network just grows independently of the dynamics. It would be quite important to adding an adaptive mechanism for the growth of the network, where, for instance, new links are constructed to support congested routes. The idea of having each pair of nodes connected by more than one link is not just equivalent to having just one link with a higher capacity (this has been studied in many publications). The reason is that add more than one link between each pair of nodes could enrich the chance of route choice between a given origin-destination (OD) pair. Moreover, adding the link to connect two nodes could increase the structure complexity of the traffic and transportation network, which means the degree distribution of network, should be different from that of the networks of the previous researches.

In this paper, a network evolving model for the traffic and transportation network is designed. In this evolving model, we consider the self-growth phenomenon during the evolving of the network. Based on the new network evolving mechanism, the node degree distribution of the generated network is deduced. From the power-law fitting results of the cumulative distribution functions (CDF) of the node degree distribution, we conclude that the traffic and transportation network owns the scale-free feature. However, the scale-free characteristic depends much on the parameters of self-growth during the evolution of the network.

\section{Evolving Model for the Traffic and Transportation Network}

Absenting from the classical random network models [1], two mechanisms are responsible for the emergence of the scaling in the exiting literatures: (1) the network evolves by adding a new node with $m\left(m \leq m_{0}\right)$ edges which links the new node to $m$ different nodes in 
the primary small network (with $m_{0}$ numbers of nodes); (2) the preferential attachment which specifies the probability that a new node will be connected to node $i$ depends on the degree of node $i$, that is, $\pi_{i}\left(k_{i}\right)=k_{i} / \sum_{j} k_{j}$. As has been stated in the first section, for the traffic and transportation network, they are commonly exited more than one link between two nodes. Furthermore, the evolution of traffic and transportation network always has the "self-growth" characteristic. Consequently, we design a new evolving model adaptive to the traffic and transportation network as follows.

Starting with a small number $\left(m_{0}\right)$ of nodes, at each time step $t=1,2, \ldots, T$, repeat the following operations $m\left(\leq m_{0}\right)$ times.

(1) Randomly select a node in the current network as the ending point of the new link, and whether node $i$ is selected as the ending point of the new link depends on its degree $k_{i}$ with the preferential probability $\pi_{i}\left(k_{i}\right)=k_{i} /\left(\sum_{j} k_{j}\right)$.

(2) Generate a random number $r(0 \leq r \leq 1)$, if $r \leq \rho$ ( $\rho$ denotes the self-growth probability for the evolution of the network), select randomly a node $j(j \neq i)$ in the system as the starting point of the new added link $(j, i)$; otherwise if $r>\rho$ : check if a new node $k$ has been added into the system, if not, add a new node $k$ to the system as the starting point and connect the ending point $i$ to the new link $(k, i)$, if yes, connect the new node $k$ to the ending point $i$ to add the new link $(k, i)$.

Apparently, at each time step $t, m$ new links can be added to the system regardless of that whether a new node is added. In order to demonstrate the growth mechanism for the traffic and transportation network, we use Figure 1 as illustration.

The primary network is shown in Figure 1(a), in which the number of links $m_{0}=4$ and the degree of each node in the system is respectively 1, 4, 2 and 1 . Assume $m=4$ and $\rho=0.5$, at time $t=1$, we should run $m(m=4)$ times of the following operations to add four links into the system.

Step 1. Based on preferential probability $\pi_{i}=k_{i} / \sum_{j} k_{j}$, select randomly a node (assume node \#2) as the ending point of new link.

Generate a random number $r$ (assume $r=0.2$ ). Since $r(=0.2) \leq \rho(=0.5)$, select randomly a node (assume node \#3) in the system as the starting point to add the new link $(3,2)$.

Step 2. Like Step 1, select randomly a node (assume node \#2) as the ending point of new link. Generate a random number $r$ (assume $r=0.7)$. Since $r(=0.7)>\rho(=0.5)$, and there has not been a new node added into the system, add a new node (node 5 ) to the system and connect it to the ending point (node 2 ) to add a new link $(5,2)$.

Step 3. Select randomly a node (assume node 4 ) as the ending point of new link. Generate a random number $r$ (assume $r=0.6$ ). Since $r(=0.6)>\rho(=0.5)$, and there has been a new node (number 5 ) in the system, connect node 5 and node 4 to add the new link $(5,4)$.

Step 4. Select randomly a node (assume node \#2) as the ending point of new link. Generate a random number $r$ (assume $r=0.65$ ). Since $r(=0.65)>\rho(=0.5)$, and there has been a new node (number 5 ) in the system, connect node 5 and node 2 to add the new link $(5,2)$.

As illustrated in Figure 1(b), we are clear about that during the evolving of the traffic and transportation network, the network could grow by adding new links between the existing nodes or adding a new node and connect it to the existing nodes. Furthermore, each pair of nodes could be connected with more than one link. Given the time period $t=1, \ldots, T$, 


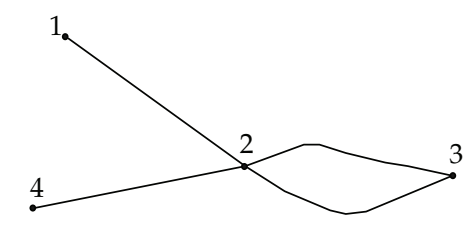

(a) $t=0$

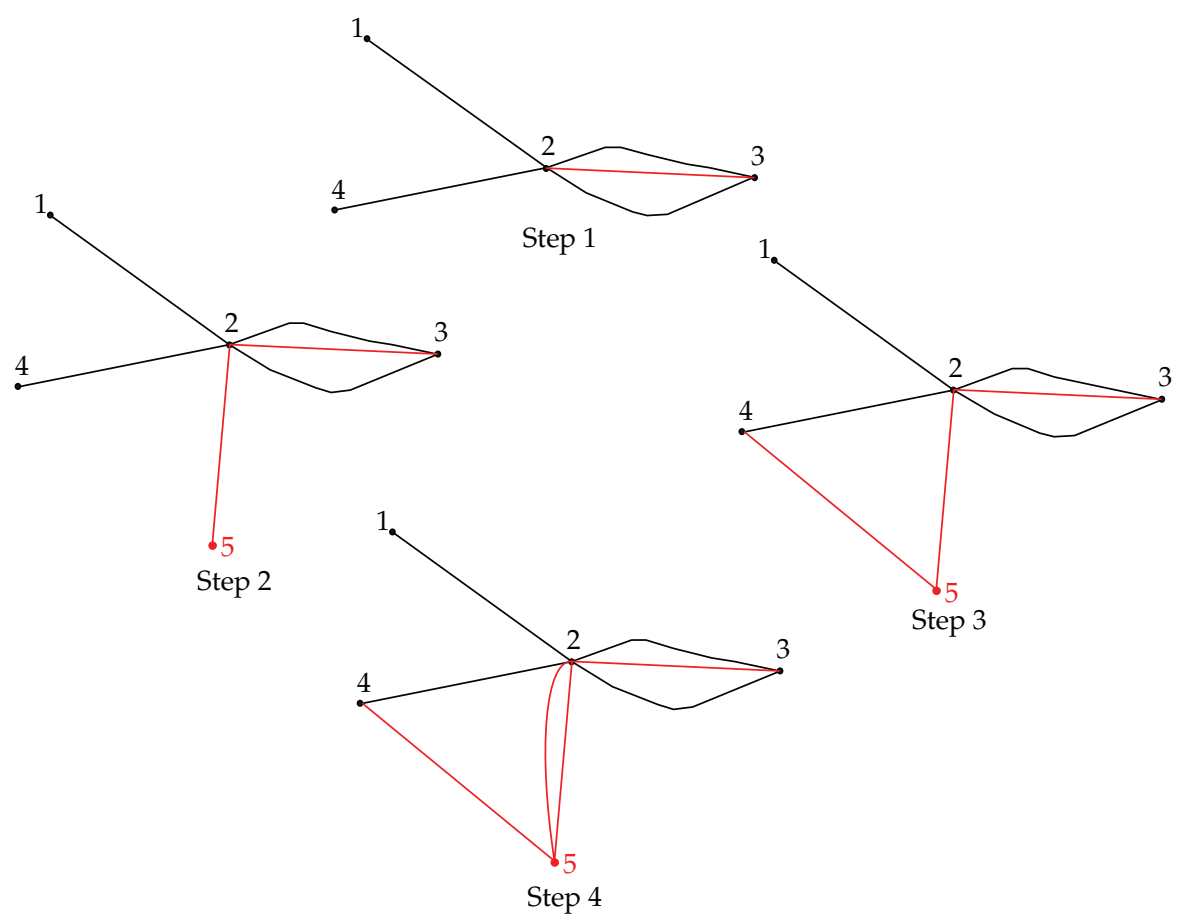

- New added link

- New added node

(b) $t=1$

Figure 1: An illustration of the network evolving mechanism for the traffic and transportation network: Assume $m_{0}=4, m=4$, and $\rho=0.5$.

as well as the self-growth probability $\rho$, we could generate the traffic and transportation network which owns the self-growth feature.

\section{Degree Distribution Model of the Traffic and Transportation Network}

According to the continuum theory, we assume that the degree of node $i$ changes continuously versus the time step $t$, denoted by $k_{i}(t)$. Let $N(t)$ represent the total number of nodes and let $L(t)$ be the total number of links in the system at time $t$. Start with $t=0, N(t=0)=m_{0}$ nodes, and $L(t=0)=l_{0}$ links. $k_{i}(t)$ satisfies the following dynamical 
equation:

$$
\begin{aligned}
\frac{\partial k_{i}}{\partial t} & =\left(1-\rho^{m}\right) m \pi_{i}\left(k_{i}\right)+\rho^{m} m\left[\pi_{i}\left(k_{i}\right)+\left(1-\pi_{i}\left(k_{i}\right)\right) \frac{1}{N(t)-1}\right] \\
& =m \pi_{i}\left(k_{i}\right)+\rho^{m} m\left[\left(1-\pi_{i}\left(k_{i}\right)\right) \frac{1}{N(t)-1}\right] \\
& =m \frac{k_{i}}{\sum_{j} k_{j}}+\rho^{m} m\left[\left(1-\frac{k_{i}}{\sum_{j} k_{j}}\right) \frac{1}{N(t)-1}\right] .
\end{aligned}
$$

In (3.1), the first term $m k_{i} /\left(\sum_{j} k_{j}\right)$ represents the probability that node $i$ is selected as the ending point of the new added link at time step $t$ in the standard BA model $[1,4]$. As stated in the above section, at each time step of the evolving of traffic and transportation network, we would run $m$ times of judging whether a new node would be added into the system. The probability that no node is added into the existing system is $\rho^{m}$ ( $\rho$ denoted as self-growth probability). So, the event that at least one node is added into the system happens with a probability of $\left(1-\rho^{m}\right)$, which multiplies $m k_{i} /\left(\sum_{j} k_{j}\right)$ to obtain the probability that node $i$ is selected as the ending point and linked to a new added node at time $t$. Similarly, the second term $\rho^{m} m\left[\left(1-k_{i} /\left(\sum_{j} k_{j}\right)\right)(1 /(N(t)-1))\right]$ represents the probability that node $i$ is selected as the starting point of the new added link in case that no node is added into the system (that is, the network undergoes a self-growth process).

According to [4], the sum of the degrees of all nodes is $\sum_{j} k_{j}=2 m t$. Accordingly, expected total number of the nodes in the system depends on the self-growth probability $\rho$, that is $N(t)=m_{0}+\left(1-\rho^{m}\right) m t$. Consequently, (3.1) could be written as

$$
\begin{gathered}
\frac{\partial k_{i}}{\partial t}=\frac{k_{i}}{2 t}+\rho^{m} m\left[\left(1-\frac{k_{i}}{2 m t}\right) \frac{1}{m_{0}+\left(1-\rho^{m}\right) m t-1}\right] \\
\approx \frac{k_{i}}{2 t}+\rho^{m} m\left[\left(1-\frac{k_{i}}{2 m t}\right) \frac{1}{\left(1-\rho^{m}\right) m t}\right], \text { for large } \mathrm{t}, \\
\frac{\partial k_{i}}{\partial t}=\frac{k_{i}}{2 t}+\frac{\rho^{m}\left(2 m t-k_{i}\right)}{2\left(1-\rho^{m}\right) m t^{2}} \\
=\frac{k_{i}}{2 t}+\frac{\rho^{m}}{\left(1-\rho^{m}\right) t}+\frac{\rho^{m} k_{i}}{2\left(1-\rho^{m}\right) m t^{2}} \\
\frac{\partial k_{i}}{\partial t} \approx \\
\approx \frac{k_{i}}{2 t}+\frac{\rho^{m}}{\left(1-\rho^{m}\right) t}, \quad \text { for large } t .
\end{gathered}
$$

The solution of (3.4), with the initial condition that node $i$ was added to the system at time $t_{i}$ with the degree $k_{i}\left(t_{i}\right)=m\left(1-\rho^{m}\right)$ (on the condition that no self-growth event has happened), is

$$
k_{i}(t)=A\left(\frac{t}{t_{i}}\right)^{1 / 2}-\frac{2 \rho^{m}}{\left(1-\rho^{m}\right)}, \text { with } A=\frac{\left(m-2 m \rho^{m}+2 \rho^{m}+m \rho^{2 m}\right)}{1-\rho^{m}}
$$


Using (3.5), the probability that a node at equal time has a degree $k_{i}$ smaller than $k$, $P\left(k_{i}(t)<k\right)$, could be expressed as:

$$
P\left(k_{i}(t)<k\right)=P\left(t_{i}>c(k) t\right)
$$

Based on (3.5) and (3.6), we have

$$
c(k)=\left(\frac{A\left(1-\rho^{m}\right)}{k\left(1-\rho^{m}\right)+2 \rho^{m}}\right)^{2} .
$$

Simply, $t_{i}$ follows a constant probability density as:

$$
P\left(t_{i}\right)=\frac{1}{m_{0}+t_{i}}
$$

Substituting this into (3.6), we could obtain

$$
P\left(t_{i}>c(k) t\right)=1-\frac{c(k) t}{m_{0}+t}=1-\frac{t}{m_{0}+t}\left(\frac{A\left(1-\rho^{m}\right)}{k\left(1-\rho^{m}\right)+2 \rho^{m}}\right)^{2} .
$$

$P(k)$ could be obtained using:

$$
\frac{\partial P\left(k_{i}(t)<k\right)}{\partial k}=\frac{\partial P\left(t_{i}>c(k) t\right)}{\partial k}=\frac{2 t}{m_{0}+t}\left(\frac{A^{2}\left(1-\rho^{m}\right)^{3}}{\left(k\left(1-\rho^{m}\right)+2 \rho^{m}\right)^{3}}\right)
$$

So that, we have:

$$
\begin{aligned}
P(k) & =\frac{\partial P\left(k_{i}(t)<k\right)}{\partial k} \sim\left[\frac{\left(m-2 m \rho^{m}+2 \rho^{m}+m \rho^{2 m}\right)}{1-\rho^{m}}\right]^{2} \frac{2\left(1-\rho^{m}\right)^{3}}{\left[\left(1-\rho^{m}\right) k+2 \rho^{m}\right]^{3}}, \quad t \rightarrow \infty \\
& =\frac{2\left(1-\rho^{m}\right)\left(m-2 m \rho^{m}+2 \rho^{m}+m \rho^{2 m}\right)^{2}}{\left[\left(1-\rho^{m}\right) k+2 \rho^{m}\right]^{3}} .
\end{aligned}
$$

From (3.11), we know that the degree distribution of the traffic and transportation network depends on the self-growth probability $\rho$. Clearly, (3.11) demonstrates that $P(k)$ follows a power-law distribution with scaling parameter (exponent gamma) near 3. Consider the following two cases:

(1) in case that $\rho \rightarrow 0$, we have $P(k) \rightarrow 2 m^{2} k^{-3}$. The evolving model is equivalent to the standard BA model [13], which has the scale-free feature according to $[1,2]$.

(2) in case that $\rho \rightarrow 1, P(k) \rightarrow 0$, which implies that the network is not growing in the way of adding new nodes at all. However, even in this case using the static model in [12], one can still yield scale-free $P(k)$. 


\section{Simulation Results}

To provide numerical support for the degree distribution function of (3.11), we generate different networks of $N=10^{4}$ nodes with initial configurations shown in Figure 1(a). We set $m=4$ versus different self-growth probabilities $\rho=0.0,0.1,0.3,0.5,0.7$, and 0.9 , respectively. After performing the network evolving model in Section $2\left(\operatorname{set} T=N=10^{4}\right)$, the node degree distribution result of each network is shown in Figure 2.

In Figure 2, we plot the histograms of event frequency versus size, event rank versus size, and event rank versus frequency of the node degree of the generated networks in panel (a) to (c) to panel (c). We also examine the log-normal behavior of the node degree distribution using the method in $[11,14]$, and. The result is shown in panel (d).

We could see that these curves on panel (a) to panel (c) display the situations of "long tails," which is the reprehensive nature of power-law distributing data set. On the other hand, as shown in panel (d) of Figure 2, it could observed that the node degree data sets own lognormal distribution behaviors in the fact that distance from means of log degree values versus square roots of logs of counts of binned values have leaner relationships according to the regression (the method used for the leaner regression could be referred in $[4,11,14]$ ). Accordingly, we could say again that our data set of node degree does have power-law distribution nature.

\section{Applications to Real-World Data}

To consolidate our result, we investigate the evolving characteristics of real traffic and transportation networks. We investigate the evolution of China domestic airline network (CDAN) from the year 1950 to 2010 and 2000 to 2010 for detailed network topology information. The node degree datasets of the networks were collected in May, 2010 from the database of the Civil Aviation Bureau of China [15].

We summarized average node degrees, number of airlines and airports (nodes) of these networks of CDAN from 1950 to 2010, which are shown in Figure 3. As described in Figure 3, we found that the average degree of networks of CDAN increased drastically from year 1950 to 2000 . However, the increase of the average node degree is relatively stationary from 2000 to 2010. The change trend of the number of airports in the network was the same as that of the average node degree. However, there was somewhat difference in change trend of the number airline of CDAN.

As shown from the green diamond line in Figure 3, we can see that the increase rate of the number of airlines is more sharply than those of the rest two items from the year 2000 to 2010. With this evidence, we could conclude that the evolution of CDAN from 2000 to 2010 obviously undergo the process of self-growth. Indeed, the number of connections between any two cities of China with the airline has increased more than 50 lines per year since 2000. On the other hand, there were only 42 new airports opened in the recent decade [15].

For a better and more complete picture of the self-growth evolution of CDAN, we plot the cumulative distribution function (CDF) of the node degree of the network of 2000, 2005, and 2010 in Figure 4. In Figure 4, we also display straight line of the fitting of power-laws of the CDF of each the node degree datasets using the MLE using in [11]. Apparently, in the three panels, the node degree data set fit the logarithmic power-law line so close that we also could know that CDAN owns the scale-free feature. From Figure 4, it could be clearly recognized that the data sequence of node degree of the network does not arrange on 


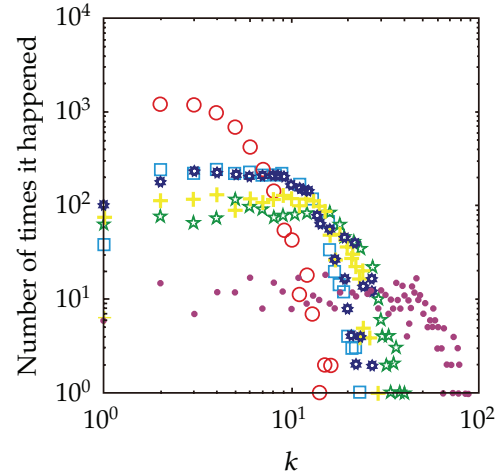

(a) Frequency vs. size plot

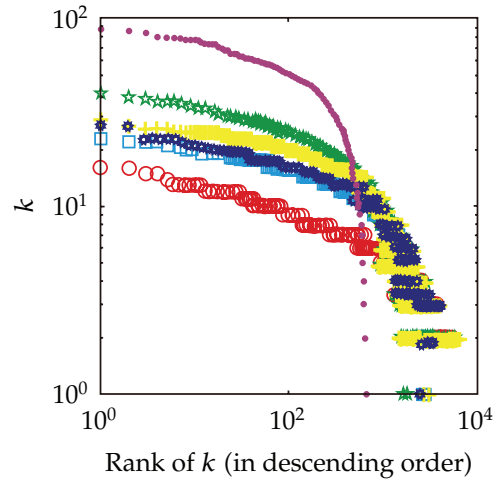

(b) Events ranked by size

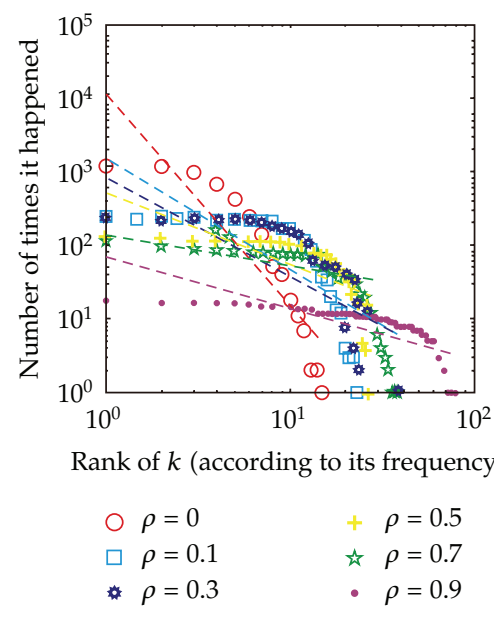

(c) Events ranked by frequency

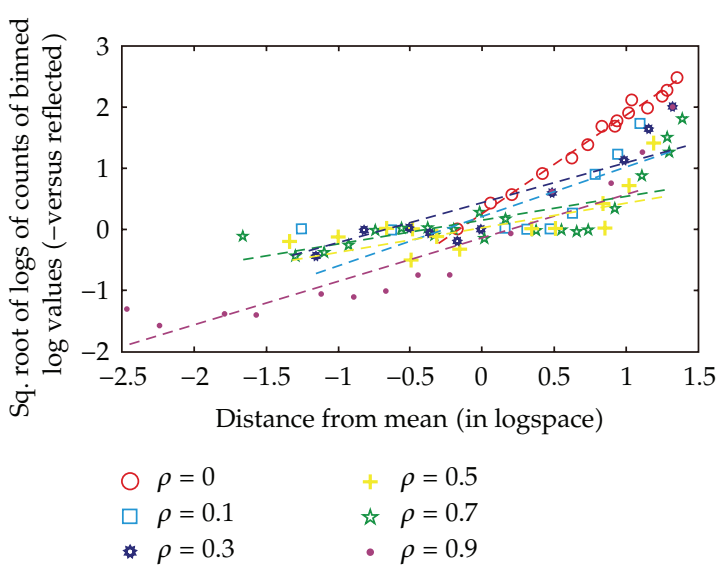

(d) Very straight (log normal)

Figure 2: Histograms of event frequency versus size, event rank versus size, event rank versus frequency and the log-normal behavior test of the node degree of networks generated with the proposed evolving model with $m=4$ versus different self-probabilities.

a straight line as the discrete power-law distributing data (which is as shown in [11]). This situation tells that the previous reports (which reported the node degree follows the pure power-law distribution, e.g., [16]) could not perfectly unfold the scaling-free feature of the airline network. As evident in Figure 4, it could be seen that, for CDAN the number of nodes with a degree more than 10 is larger and larger from year 2000 to 2010. This situation implies that the network has evolved mainly in the way of connecting the existing airports with the newly opened airlines from year 2000 to 2010, rather than opening the new airport.

Since the data node degrees of CDAN from 2000 to 2010 has been approved following the power-law distribution. For measuring the qualities of these distributions, we test the power-law hypothesis using the methods described in [11]. The relevant results are summarized in Table 1, in which: $\widehat{\alpha}$ denotes the estimated value of the scaling parameters of power-law distribution; $\sigma_{\widehat{\alpha}}$ is the standard error on $\widehat{\alpha} ;\langle k\rangle$ is the average node degree as mentioned before; $k_{\min }$ is the smallest node degree.

As shown in Table 1, we could see in the second column that the estimated scaling parameters $\widehat{\alpha}$ of power-law distribution of the node degree of CDAN decreases gradually 


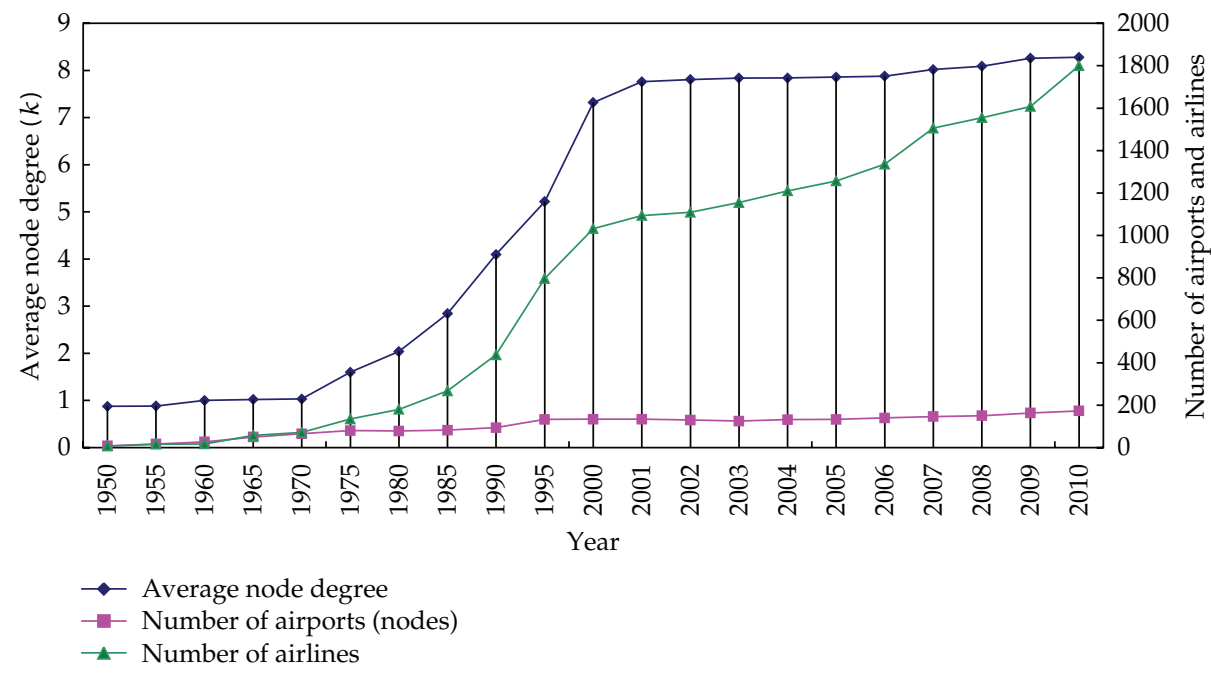

Figure 3: Average node degree and the number of airports/airlines of the China domestic airline network from year 1950 to 2010 .

Table 1: Basic parameters of power-law distribution tests of China domestic airline networks, from year 2000 to 2010.

\begin{tabular}{llccc}
\hline Year & $\langle k\rangle$ & $\widehat{\alpha}$ & $\sigma_{\widehat{\alpha}}$ & $k_{\min }$ \\
\hline 2000 & 7.26 & 3.09 & 3.12 & 1.00 \\
2001 & 7.32 & 3.15 & 4.45 & 1.00 \\
2002 & 7.76 & 3.21 & 6.51 & 1.00 \\
2003 & 7.81 & 2.45 & 6.75 & 1.00 \\
2004 & 7.84 & 2.35 & 7.18 & 1.00 \\
2005 & 7.84 & 2.46 & 8.52 & 1.00 \\
2006 & 7.86 & 2.33 & 8.19 & 1.00 \\
2007 & 7.88 & 2.22 & 9.62 & 1.00 \\
2008 & 8.02 & 2.11 & 11.25 & 1.00 \\
2009 & 8.09 & 2.15 & 17.01 & 1.00 \\
2010 & 8.26 & 2.12 & 19.15 & 1.00 \\
\hline
\end{tabular}

from 2000 to 2010. Instead, the standard error on $\widehat{\alpha}$ increases bit by bit from 2000 to 2010 . This phenomenon could be explained by the self-growth nature of the airline network. From year 2000 to 2010, the self-growth probability of the network might be relevantly small initially. Consequently, the estimated scaling parameter of the power-law distribution is closed to 3.0 with a small standard error. However, as the self-growth probability increases, the estimated scaling parameter of power-law distribution as well as its standard error is heavily influenced by the self-growth situation. This situation could also be interpreted using (3.11). If the selfgrowth probability $\rho$ is large enough and closed to 1.0, the node degree distribution of the network might not follow the pure power-law distribution. That is the estimated scaling parameter of power-law distribution would be smaller than 3.0 and have the larger standard error.

Comparing with the published works, it was reported in $[10,17,18]$ that traffic and transportation networks followed power-law distributions and hold a scaling parameter 


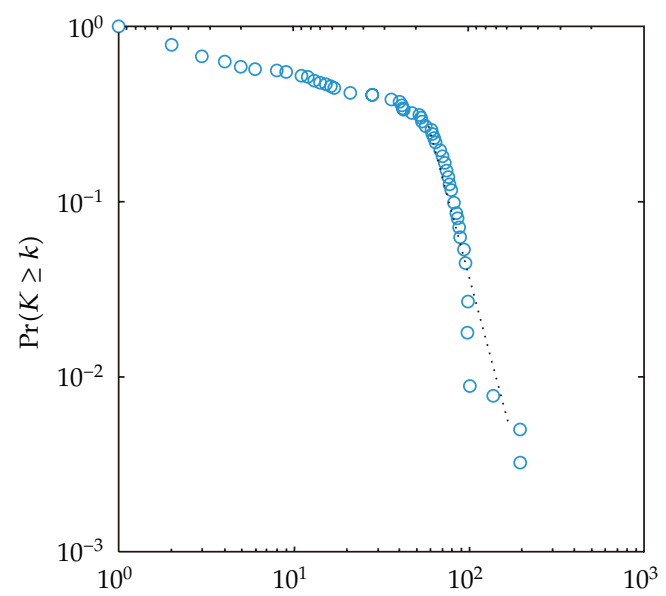

(a)

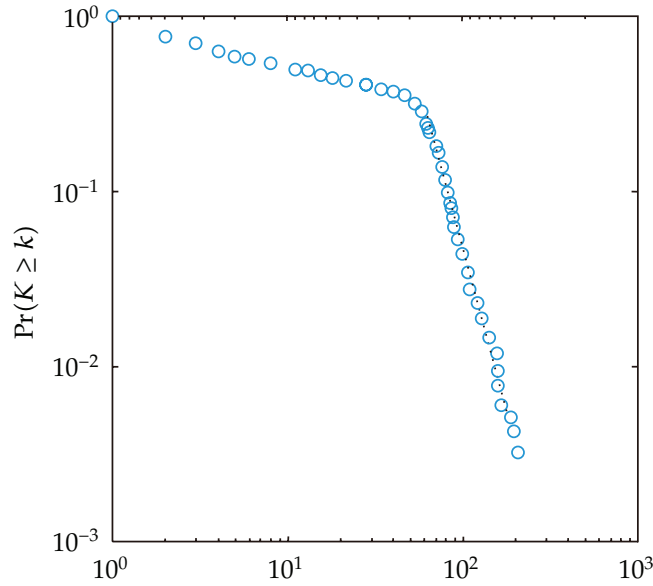

(b)

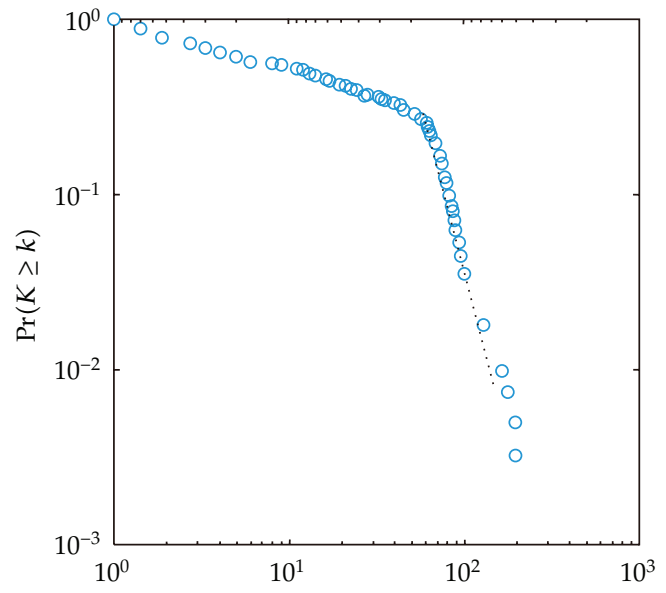

(c)

Figure 4: The cumulative distribution function $\operatorname{Pr}(K)$ and their straight line power-law fits of the node degree of China Domestic airline network at year 2000, 2005, 2010.

range from 1.0 to 2.4. Here, we approve that CDAN is scale-free but follows a power-law distribution with a scaling parameter between 3.0 with the varied standard error, which is constrained by the self-growth parameters during the evolution of the airline network.

\section{Conclusions}

The study of the structure of the traffic the transportation network from the perspective of complex network has drawn considerable researching attention. Unfortunately, the opinions of the previous researchers are identical that they take account of the evolution of the traffic and transportation network without considering the situation of self-growth. In this paper we proposed an evolving model which is relevantly adaptive for the traffic and transportation network. According to the analytic results for node degree distributions of the tested networks generated via the proposed model, we find that the networks are scale-free 
due to that their node degree distributions follow the power-law distributions. We also test the distribution of the node degree of the real traffic and transportation network and conclude that our evolving model is more appropriate to explain the scale-free feature as well as the self-growth situation of the traffic and transportation network.

We hope that the methods given in this paper could provide useful evidences for determining the optimal structure of the traffic and transportation network, which also is crucial to our future research. Geographic or spatial networks, such as an urban street network or railway network, have strong constraints during their growth due to the fact that their nodes and edges are embedded in time and space. This fact should also be explicitly investigated in the future research.

\section{Acknowledgments}

This work is supported by National Natural Science Foundation of China under Grant no. 71171171. The authors would like to thank anonymous reviewers for their insightful comments and suggestions.

\section{References}

[1] A.-L. Barabási and R. Albert, “Emergence of scaling in random networks," Science, vol. 286, no. 5439, pp. 509-512, 1999.

[2] D. J. Watts and S. H. Strogatz, "Collective dynamics of "small-world" networks," Nature, vol. 393, no. 6684, pp. 440-442, 1998.

[3] A. L. Barabási and Z. N. Oltvai, "Network biology: understanding the cell's functional organization," Nature Reviews Genetics, vol. 5, no. 2, pp. 101-113, 2004.

[4] S. Boccaletti, V. Latora, Y. Moreno, M. Chavez, and D.-U. Hwang, "Complex networks: structure and dynamics," Physics Reports, vol. 424, no. 4-5, pp. 175-308, 2006.

[5] A. Barrat, M. Barthélemy, R. Pastor-Satorras, and A. Vespignani, "The architecture of complex weighted networks," Proceedings of the National Academy of Sciences of the United States of America, vol. 101, no. 11, pp. 3747-3752, 2004.

[6] R. Guimerà, S. Mossa, A. Turtschi, and L. A. N. Amaral, "The worldwide air transportation network: anomalous centrality, community structure, and cities' global roles," Proceedings of the National Academy of Sciences of the United States of America, vol. 102, no. 22, pp. 7794-7799, 2005.

[7] W. Wang, Y. Mao, J. Jin et al., "Driver's various information process and multi-ruled decisionmaking mechanism: a fundamental of intelligent driving shaping model," International Journal of Computational Intelligence Systems, vol. 4, no. 3, pp. 297-305, 2011.

[8] J. J. Wu, Z. Y. Gao, and H. J. Sun, "Model for dynamic traffic congestion in scale-free networks," Europhysics Letters, vol. 76, no. 5, p. 787, 2006.

[9] B. Tadić, S. Thurner, and G. J. Rodgers, "Traffic on complex networks: towards understanding global statistical properties from microscopic density fluctuations," Physical Review E, vol. 69, no. 3, Article ID 036102, 5 pages, 2004.

[10] H. Sun, J. Wu, and Z. Gao, "Dynamics of traffic networks: from microscopic and macroscopic perspectives," Physica A, vol. 387, no. 7, pp. 1648-1654, 2008.

[11] A. Clauset, C. R. Shalizi, and M. E. J. Newman, "Power-law distributions in empirical data," SIAM Review, vol. 51, no. 4, pp. 661-703, 2009.

[12] K. I. Goh, B. Kahng, and D. Kim, "Universal behavior of load distribution in scale-free networks," Physical Review Letters, vol. 87, no. 27, Article ID 278701, 4 pages, 2001.

[13] M. Barthélemy, "Spatial networks," Physics Reports, vol. 499, no. 1-3, pp. 1-101, 2011.

[14] S. Milojević, "Power law distributions in information science: making the case for logarithmic binning," Journal of the American Society for Information Science and Technology, vol. 61, no. 12, pp. 24172425, 2010.

[15] China and C. A. A. O., Statistical Data on Civil Aviation of China 1950-2010, Chinese Civil Aviation Press, 2010. 
[16] L. A. N. Amaral, A. Scala, M. Barthélémy, and H. E. Stanley, “Classes of small-world networks," Proceedings of the National Academy of Sciences of the United States of America, vol. 97, no. 21, pp. 1114911152, 2000.

[17] R. V. Donner, J. Heitzig, J. F. Donges, Y. Zou, N. Marwan, and J. Kurths, "Geometric detection of coupling directions by means of inter-system recurrence networks," Physics Letters A, vol. 373, no. 46, pp. 4246-4254, 2009.

[18] H. Jeong, "Complex scale-free networks," Physica A, vol. 321, no. 1-2, pp. 226-237, 2003. 


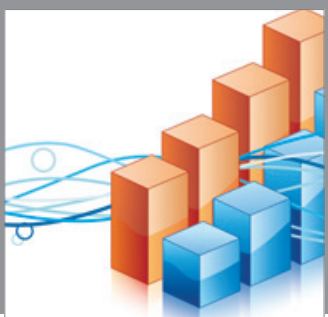

Advances in

Operations Research

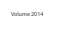

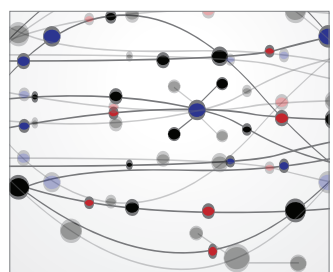

\section{The Scientific} World Journal
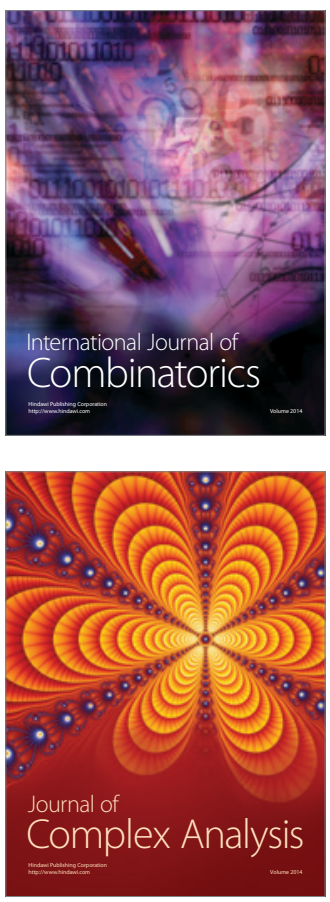

International Journal of

Mathematics and

Mathematical

Sciences
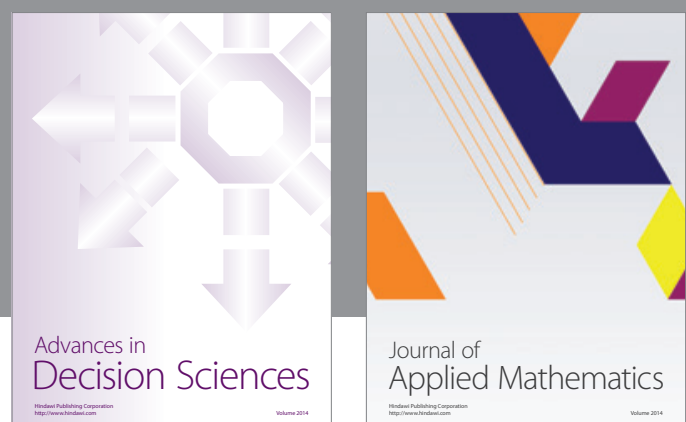

Journal of

Applied Mathematics
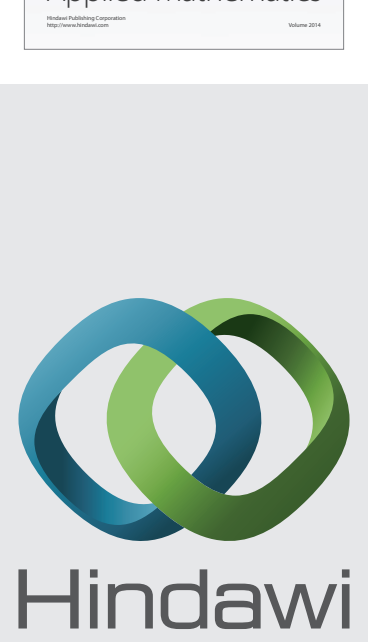

Submit your manuscripts at http://www.hindawi.com
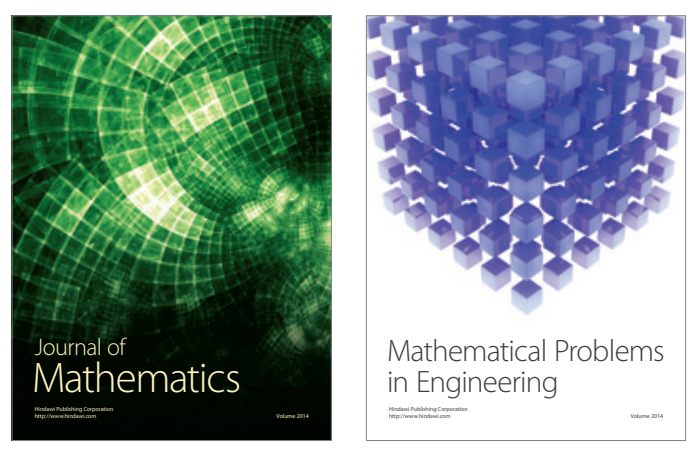

Mathematical Problems in Engineering
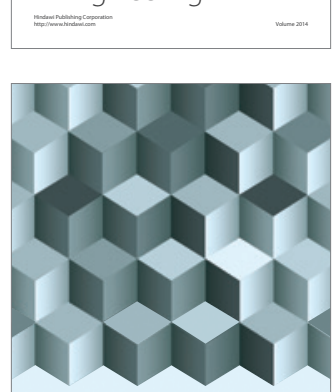

Journal of

Function Spaces
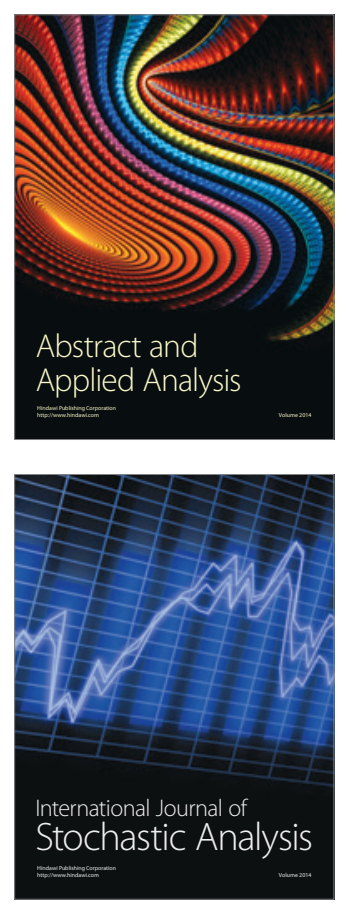

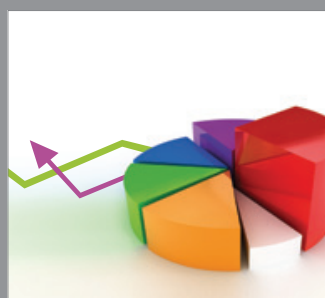

ournal of

Probability and Statistics

Promensencen
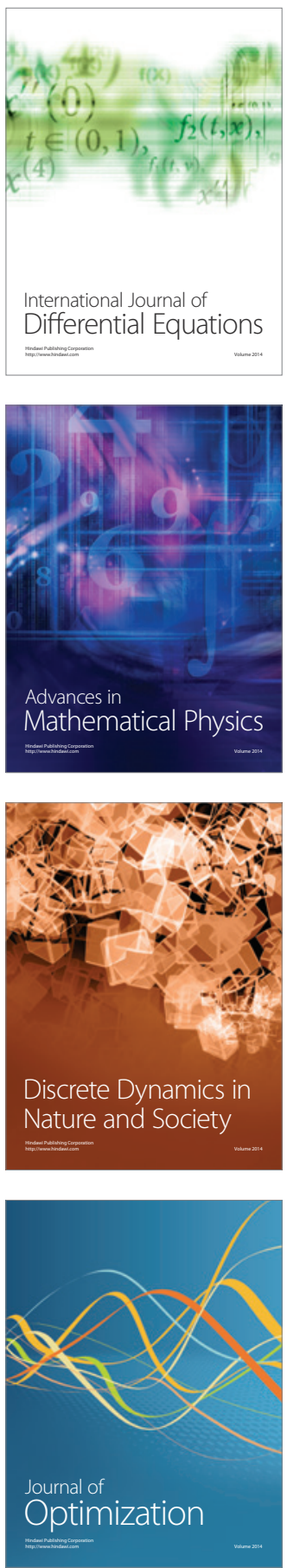